\title{
Disappearance of Pulmonary Cavity caused by Fungal Infection
}

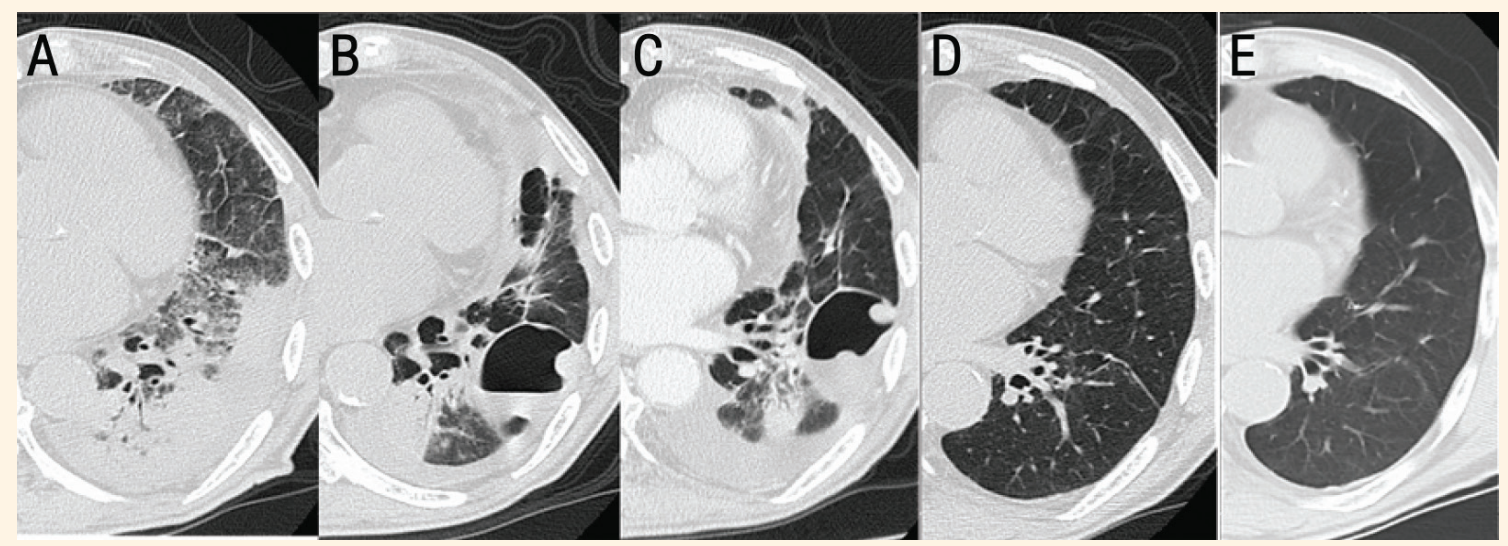

Figure 1: Chest computed tomography of a 79-year-old male patient showing changes until the disappearance of the cavity in left lower lobe of the lung. A: Consolidation in left lung with no cysts or cavities in the left lung at admission. B: Appearance of a cavity with a spherical structure suspected to be a 'fungus ball' or blood clot taken 2 weeks after the initiation of antifungal treatment. C: Cavity that remained unchanged in shape and size after 12 weeks of administration of voriconazole. D: Change from cavity to linear shadow 12 months after the diagnosis. E: Disappearance of linear shadow 18 months after the diagnosis.

\section{$\mathrm{T}$} He disappearance of CAVities associated with pulmonary fungal infections is rare. ${ }^{1} \mathrm{~A}$ 79-year-old male patient was admitted to a local hospital in Mito-city, Japan, in 2017 complaining of cough and fever for the previous two weeks. The patient had no history of diabetes or tuberculosis. At admission, a blood analysis showed a white blood cell count of $13,600 / \mu \mathrm{L}, \mathrm{C}$-reactive protein of 12.37 $\mathrm{mg} / \mathrm{dL}$, serum creatinine of $1.27 \mathrm{mg} / \mathrm{dL}$ and blood glucose of $206 \mathrm{mg} / \mathrm{dL}$. Chest computed tomography (CT) scan taken at admission showed consolidation in the left lung, but no cysts or cavities were observed [Figure 1A]. Cefepime was administered for three days but his symptoms worsened. Considering the antibacterial spectrum, cefepime was changed to micafungin (100 mg/day). Aspergillus antigen test was negative but plasma $\beta$-D-glucan was found to be $118 \mathrm{pg} / \mathrm{mL}$ (normal range: $0-20 \mathrm{pg} / \mathrm{mL}$ ). Although no pathogen grew in the culture of bronchial lavage fluid, septate hyphae with branching at 45-degree angles were observed, suggestive of Aspergillus infection. ${ }^{2}$ Micafungin was changed to oral voriconazole (300 $\mathrm{mg} /$ day) and intravenous liposomal amphotericin B (150 mg/day) was added and continued for three weeks. The cough and fever as well as inflammatory indices improved. However, the appearance of a cavity in the chest was found by CT taken two weeks after the initiation of antifungal treatment. In this scan, a spherical structure suspected to be a 'fungus ball' or blood clot was found in the cavity [Figure 1B]. Oral voriconazole was administered for 12 weeks but the cavity remained unchanged [Figure 1C]. By 12 months, however, the cavity changed to linear shadow by $\mathrm{CT}$ [Figure 1D], and at 18 months, it was not observed by CT [Figure 1E]. The patient was well 37 months after this infection.

This report conformed to the Ethical Guidelines for Clinical Studies issued by the Ministry of Health, Labor and Welfare in Japan. Written comprehensive consent was obtained from the patient. Reporting of this case report was approved by the Ethics Committee in the presenting hospital (NO16-66).

\section{Comments}

Pulmonary fungal infections are often refractory because they usually occur in compromised hosts.,4 In patients with pulmonary mycosis, some develop 
cavities. ${ }^{5}$ Once formed, they often change to linear shadows or remain as cavities but rarely disappear. ${ }^{1}$ The current elderly patient had impaired glucose tolerance; in addition, the patient was diagnosed with left renal cancer one year after this infectious episode. It was unclear whether the impaired glucose tolerance and cancer-bearing status in this patient were associated with the fungal infection. To the best of the authors' knowledge, there is one report of a patient with pulmonary fungal infection who followed a similar course until the disappearance of a lung cavity. ${ }^{1}$ This was a 26-year-old man with pulmonary aspergillosis, who had bronchial asthma as the underlying disease. ${ }^{1}$ Since it was a pulmonary mycosis that developed in a compromised elderly patient, it was speculated that the cavity would remain after treatment. The patient could be followed-up for a long period of time, even after the inflammatory response had improved and the disappearance of the cavity was unexpectedly confirmed. The cavities formed by fungal infections usually remain and the residual cavities are often not followed for long periods of time after symptoms and the inflammatory response have improved. Rare reports of cavity disappearance may be related to this. Although the mechanism is unknown, cavities formed by fungal infection may disappear as observed in the current patient.

\section{AUTHORS' CONTRIBUTIONS}

$\mathrm{KN}$, YS and HS collected the data. KN and HS prepared the manuscript. All authors approved the final version of the article.

\section{References}

1. Saitou M, Suzuki T, Niitsuma K. Multiple pulmonary Aspergillus fumigatus cysts and cavities that disappeared with anti-fungal agents. Respirol Case Rep 2018; 6:e00327. https://doi.org/10.10 $02 /$ rcr2.327

2. Kradin RL, Mark EJ. The pathology of pulmonary disorders due to Aspergillus spp. Arch Pathol Lab Med 2008; 132:606-14. https://doi.org/10.5858/2008-132-606-TPOPDD.

3. Giacobbe DR, Riccardi N, Vena A, Bassetti M. Mould infections of traumatic wounds: A brief narrative review. Infect Dis Ther 2020; 9:1-15. https://doi.org/10.1007/s40121-020-00284-8.

4. Azoulay E, Russell L, Van de Louw A, Metaxa V, Bauer P, Povoa P, et al. Diagnosis of severe respiratory infections in immunocompromised patients. Intensive Care Med 2020; 46:298-314. https://doi.org/10.1007/s00134-019-05736-5.

5. Ketai L, Currie BJ, Holt MR, Chan ED. Radiology of chronic cavitary infections. J Thorac Imaging 2018; 33:334-43. https:// doi.org/10.1097/RTI.0000000000000346. 